Journal of the Operations Research

Society of Japan

Vol. 39, No. 3, September 1996

\title{
BOND PORTFOLIO OPTIMIZATION PROBLEMS AND THEIR APPLICATIONS TO INDEX TRACKING: A PARTIAL OPTIMIZATION APPROACH
}

\author{
Hiroshi Konno Hidetoshi Watanabe \\ Tokyo Institute of Technology
}

(Received March 1, 1994; Final October 25, 1995)

\begin{abstract}
We will discuss exact and efficient parametric simplex algorithms for solving a class of nonconvex minimization problems associated with bond portfolio optimization models which one of authors proposed in the late 1980's. We will show that globally optimal solutions of both total and partial optimization problems can now be calculated on a real time basis. Also we will present some computational results of a partial optimization model applied to a tracking of an index portfolio.
\end{abstract}

\section{Introduction}

In the late 1980's, one of the authors proposed a pair of bond portfolio optimization models taking into account a variety of objectires and constraints associated with the dealing activities of bond managers of institutional investors. [7].

Of the two models, one is the "total" optimization model which intends to optimize (either maximize or minimize) a certain index associated with a total portfolio after a transaction (Fig 1(a)). This model was formulated as a bilinear fractional programming problem, whose good locally optimal solution (which may or may not be a globally optimal solution) can be calculated reasonably fast by a heuristic algorithm based upon the simplex method for linear programming problems. Hence, this model has been used in practice by several institutional investors both in Japan and in U.S..

The "partial" optimization model, on the other hand tries to optimize the difference of a given index associated with the bundle of assets purchased from the market and sold to the market (Fig 1(b)). Though odd looking from a viewpoint of standard approach in portfolio optimization, partial optimization is often considered more attractive from the viewpoint of practitioners as evidenced by a series of interviews with a number of bond managers of institutional investors. The primary reason is that only a small fraction, typically less than $10 \%$ of the total assets owned by an investor is sold and/or purchased in a typical transaction.

Therefore the resulting improvement of the objectire function before and after a transaction in terms of total optimization model is very small since at least $90 \%$ of the assets remains the same, while the improvement in terms of partial optimization model can be very significant. In fact, the competence of a bond manager is appreciated more in terms of partial optimization than total optimization.

Unfortunately, however this partial optimization model results in a difficult nonconvex minimization problem, for which the author could not devise an exact and efficient algorithm in [7] and thus this model was not used in practice until recently. 


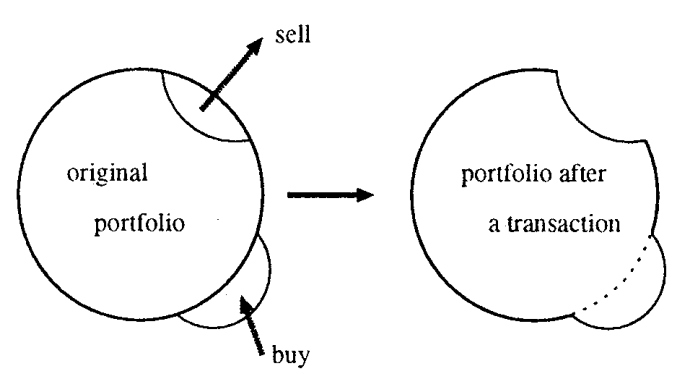

(a) Total Optimization Model

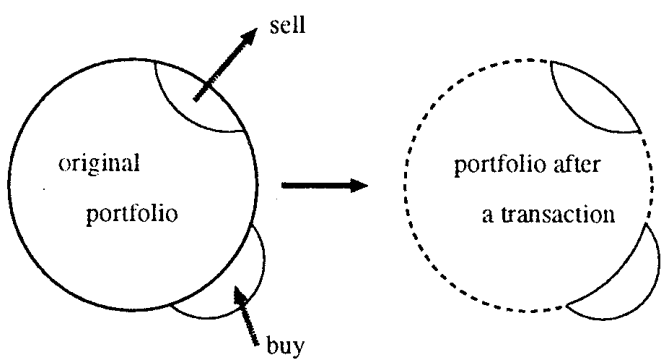

(b) Partial Optimization Model

Figure 1: Bond Portofolio Optimization Models

The purpose of this paper is to demonstrate that this nonconvex minimization problem resulting from the partial optimization model can be solved to global optimality very fast as a result of recent derelopments in global optimization methods [6]. In fact they can be solved in approximately the same amount of computation time as that needed for solving a linear programming problem of the same size. This means that we can solve both total and partial optimization models on a real time basis.

The readers will find that the model developed in this paper is different from the classical and standard bond portfolio optimization models in which the portfolio is constructed so as to meet the (fixed) future cash flows with minimal cost under the assumption that the investor holds the portfolio until maturity. (The readers are referred to the standard text such as Elton-Gruber [4] and Fong-Fabozzi [5], for conventional models.

To the contrary, the model developed in this paper is concerned with the dealing of bonds where the portfolio manager tries to hold a portfolio with maximal performance index in an effort to maximize his or her profit. We believe that this kind of model would play more and more important roles in bond dealing business and bond management associated with asset allocation.

In the next section, we will explain several basic notions associated with bond portfolio management. In section 3, we will introduce total and partial optimization models. Section 4 will be deroted to exact and fast algorithms for solving these models which is based upon a variant of parametric simplex algorithm [9] for solving a class of global optimization problems. Finally in Section 5, we show some preliminary results of our numerical experiments using the historical data of gorernment bonds available in the market, Here we will concentrate on the application of partial optimization model to index tracking.

\section{Bond Portfolio Optimization Models}

Let us assume that an investor holds $u_{j}$ units of bonds $B_{j}(j=1, \ldots, n)$. Associated with $B_{j}$ are four basic attributes[4.5.7].

$c_{j}$ : coupon to be paid at a fixed rate (yen/bond/year)

$f_{j}$ : principal value to be refunded at maturity (yen/bond)

$p_{j}$ : unit transaction price in the market (yen/bond)

$t_{j}$ : maturity (number of years until the principal ralue is refunded)

It is well known that if the interest rate $i$ remains constant throughout the life of a bond, then the theoretical price $p_{j}$ of $B_{j}$ is giren by the following formula: 


$$
p_{j}=\sum_{t=1}^{t_{j}} \frac{c_{j}}{(1+i)^{t}}+\frac{f_{j}}{(1+i)^{t_{j}}}
$$

To emphasize the dependence of $p_{j}$ on the interest rate $i$. we emplor an altemative notation $p_{j}(i)$ in the sequel. The duration and convexity of $B_{j}$ are then defined as follows:

$$
\begin{aligned}
d_{j}(i) & =-p_{j}^{\prime}(i) / p(i) \\
C_{j}(i) & =p_{j}^{\prime \prime}(i) / p(i)
\end{aligned}
$$

In reality howerer, the interest rate raries from period to period. Under such circumstances, the theoretical price of bond (1) has to be replaced by taking its fluctuation into account. Let $T=\max _{1 \leq j \leq n} t_{j}$ and let $c_{j t}$ be the cash flow from $B_{j}$ cluring period $t$. Then the theoretical bond price is given by

$$
p_{j}\left(i_{1}, \ldots, i_{t_{j}}\right)=\frac{c_{j_{1}}}{\left(1+i_{1}\right)}+\frac{c_{j_{2}}}{\left(1+i_{1}\right)\left(1+i_{2}\right)}+\cdots+\frac{c_{j_{T}}}{\left(1+i_{1}\right)\left(1+i_{2}\right) \cdots\left(1+i_{T}\right)}
$$

where $i_{t}$ is the interest rate to be applied during period $t(t=1, \ldots T)$. Also the churation $d_{j}$ and $C_{j}$ has to be replaced by

$$
\begin{aligned}
& d_{j}\left(i_{1} \ldots, i_{T}\right)=-p_{j}^{\prime}\left(i_{1}, \ldots i_{T}\right) / p_{j}\left(i_{1} \ldots i_{T}\right) \\
& C_{j}\left(i_{1} \ldots i_{T}\right)=p_{j}^{\prime \prime}\left(i_{1} \ldots, i_{T}\right) / p_{j}\left(i_{1} \ldots, i_{T}\right)
\end{aligned}
$$

where

$$
\begin{aligned}
& p_{j}^{\prime}\left(i_{1}, \ldots i_{T}\right)=\lim _{\Delta i-0} \frac{p_{j}\left(i_{1}+\Delta i \ldots i_{T}+\Delta i\right)-p_{j}\left(i_{1}, \ldots, i_{T}\right)}{\Delta i} \\
& p_{j}^{\prime \prime}\left(i_{1}, \ldots i_{T}\right)=\lim _{\Delta i-0} \frac{p_{j}^{\prime}\left(i_{1}+\Delta i, \ldots i_{T}+\Delta i\right)-p_{j}^{\prime}\left(i_{1}, \ldots i_{T}\right)}{\Delta i}
\end{aligned}
$$

Let us note that the term structure $\left(i_{1}, \ldots, i_{T}\right)$ can be calculated very fast by using several efficient methods including[8].

Bond managers of institutional investors, when evaluating a portfolio $u=\left(u_{1}, \ldots, u_{n}\right)$ take into account such performance indices as:

(a) arerage unit price $\pi(u)$

$$
\pi(u)=\sum_{j=1}^{n} p_{j} u_{j} / \sum_{j=1}^{n} u_{j}
$$

(b) average direct vield (arerage coupon rate) $c(u)$

$$
c(u)=\sum_{j=1}^{n} c_{j} u_{j} / \sum_{j=1}^{n} u_{j}
$$

(c) average maturity $t(u)$

$$
t(u)=\sum_{j=1}^{n} t_{j} u_{j} / \sum_{j=1}^{n} u_{j}
$$

(d) average duration $d(u)$

$$
d(u)=\sum_{j=1}^{n} d_{j} p_{j} u_{j} / \sum_{j=1}^{n} p_{j} u_{j}
$$

(e) arerage convexity $C(u)$

$$
C(u)=\sum_{j=1}^{n} C_{j} p_{j} u_{j} / \sum_{j=1}^{n} p_{j} u_{j}
$$


Most fund managers prefer to have a portfolio with larger arerage unit price and arerage direct yield. Also many; if not all managers, prefer to have a shorter arerage maturity. because a larger risk is associated with a portfolio with a longer maturity. Further they prefer to have average duration and average convexity to remain within a certain interval to aroid risk associated with the fluctuation of interest rates.

In addition to five indices listed above, most fund managers want to have larger average yield to maturity, to be defined below. The traditional definition of yield to maturity $\nu_{j}$ of a bond $B_{j}$ is the smallest nonnegative solution of the following nonlinear equation:

$$
p_{j}(1+\xi)^{t_{j}}=\sum_{t=1}^{t_{j}} c_{j}(1+\xi)^{t}+f_{j}
$$

where $p_{j}$ is the price of $B_{j}$ in the market. Instead. we will employ an alternative (and more meaningful) definition:

$$
p_{j}\left(1+\nu_{j}\right)^{t_{j}}=\sum_{t=1}^{t_{j}} c_{j}\left(1+i_{1}\right) \cdots\left(1+i_{t}\right)+f_{j}
$$

The left hand side is the total amount of cash obtained by saving $p_{j}$ at the compound annual interest rate $\nu_{j}$ until maturity while the right hand side is the total amount of cash obtained by saring all coupon payments under the interest rate structure $i_{t}\left(t=1, \ldots t_{j}\right)$ plus the principal value. The average effective yield $\nu(u)$ of a portfolio $u$ is defined as follows:

(f) Average effective yield $\nu(u)$

$$
\nu(u)=\sum_{j=1}^{n} \nu_{j} p_{j} u_{j} / \sum_{j=1}^{n} p_{j} u_{j}
$$

It is easy to see that the trader will get the effective yield $\nu(u)$ per period by loolding a portfolio $\left(u_{1}, \ldots, u_{n}\right)$. Thus he or she prefers to have larger average effective yield.

\section{Mathematical Description of Bond Portfolio Optimization Models.}

Let us assume again that an investor holds $u_{j}$ units of $B_{j},(j=1, \ldots, n)$ out of which $n_{1}$ are chosen as candidates for sale in the market. In a typical situation, $n$ is a few hundred and $n_{1}$ is less than one hundred. Also let us assume that $U_{k}$ units of bond $B_{k}^{\prime}\left(k=1, \ldots, n_{2}\right)$ are available in the market. The bond trader sells $B_{j}\left(j=1, \ldots, n_{1}\right)$ to the market and purchases $B_{k}^{\prime}$ from the market to improve a portfolio. In a typical situation, he chooses a particular index out of (a) (f) explained in Section 2 and tries to optimize (either maximize or minimize) it while putting others into constraints by specifying the least desirable level for each of them.

Let $x_{j}=$ amount of $B_{j}$ to be sold, $j=1, \ldots, n_{1}$

$$
X_{k}=\text { amount of } B_{k}^{\prime} \text { to be purchased, } k=1, \ldots, n_{2}
$$

These variables have to satisfy the conditions:

$$
\begin{aligned}
& 0 \leq x_{j} \leq u_{j}, j=1 \ldots, n_{1} \\
& 0 \leq X_{k} \leq U_{k}, k=1 \ldots, n_{2}
\end{aligned}
$$

In addition to these, several constraints are associated with transactions such as the restrictions on the total amount of bonds to be sold and/or purchased, total amount of profit and/or loss and the total amount of liquidation. all of which are represented as a linear function of $x_{j}$ 's and $x_{k}$ 's.

Let us note that all of indices $(a) \sim(f)$ as well as the constraints above belong to a class of linear or bilinear fractional functions of $u$ if the price $p_{j}$ 's are constants. Unfortunately. horrever some of $p_{j}$ is are rariables rather than constants in a typical transaction enrironment. When a bond trader simultaneously sells and buys bonds through a same agent, he is 
entitled to choose the price of each bond within a certain interral provided the agent agrees to this transaction. The reason why a trader agrees to sell a bond $B_{j}$ for the price lower than the market price $p_{j}$ is that he wants to reduce the amount of profit (difference between selling price $p_{j}$ and the book price $p_{j_{0}}$ ) out of this transaction. thereby reduce the amount of tax. The agent may instead agree to sell another bond, $B_{k}^{\prime}$ for the price lower than the market price $P_{k}$ to compensate a loss of the trader. The transaction price is, however not permitted to deviate more than a few percent from the reference price due to a regulation imposed by the government.

Let $y_{i}$ and $Y_{k}$ be the unit transaction price of $B_{j}$ and $B_{k}^{\prime}$. respectively. They have to satisfy

$$
\begin{aligned}
& \left(1-\lambda_{j}\right) p_{j} \leq y_{j} \leq\left(1+\lambda_{j}\right) p_{j}, j=1, \ldots, n_{1} \\
& \left(1-\lambda_{k}^{\prime}\right) P_{k} \leq Y_{k} \leq\left(1+\lambda_{k}^{\prime}\right) P_{k}, k=1, \ldots, n_{2}
\end{aligned}
$$

where $p_{j}$ and $P_{k}$ are the price of $B_{j}$ and $B_{k}^{\prime}$, respectively in the market and $\lambda_{j}$ and $\lambda_{k}^{\prime}$ are constants called "price adjustment coefficients". Thus a generic total optimization model [i] can be formulated as follows:

$$
(P) \mid \begin{array}{ll}
\text { maximize } & \frac{\sigma_{0}-\sum_{j=1}^{n_{1}}\left(q_{j}+q_{j}^{\prime} y_{j}\right) x_{j}+\sum_{k=1}^{n_{2}}\left(Q_{k}+Q_{k}^{\prime} Y_{k}\right) X_{k}}{\pi_{0}-\sum_{j=1}^{n_{1}}\left(r_{j}+r_{j}^{\prime} y_{j}\right) x_{j}+\sum_{k=1}^{n_{2}}\left(R_{k}+R_{k}^{\prime} Y_{k}\right) X_{k}} \\
\text { subject to } \quad \beta_{i} \leq \frac{\phi_{0}-\sum_{j=1}^{n_{1}}\left(f_{i j}+f_{i j}^{\prime} y_{j}\right) x_{j}+\sum_{k=1}^{n_{2}}\left(F_{i k}+F_{i k}^{\prime} Y_{k}\right) X_{k}}{\psi_{0}-\sum_{j=1}^{n_{1}}\left(d_{i j}+d_{i j}^{\prime} y_{j}\right) x_{j}+\sum_{k=1}^{n_{2}}\left(D_{i k}+D_{i k}^{\prime} Y_{k}\right) X_{k}} \leq a_{i}, \\
\\
\\
\sum_{j=1}^{n_{1}} a_{l j} x_{j}+\sum_{k=1}^{n_{2}} a_{l k}^{\prime} X_{k} \leq a_{l 0}, l=1, \ldots, m_{2} \\
0 \leq x_{j} \leq u_{j}, y_{j}^{0} \leq y_{j} \leq y_{j}^{1}, j=1, \ldots, n_{1} \\
0 \leq X_{k} \leq U_{k}, Y_{k}^{0} \leq Y_{k} \leq Y_{k}^{1}, k=1, \ldots, n_{2}
\end{array}
$$

This model would serve as a reference model when a significant portion, say more than one third of the assets owned by an investor is subject to sale.

Let us note that we can assume without loss of generality that the divisors of the fractional terms of the objective function and constraints are positive for all solutions satisfying other constraints. Therefore the problem $(P)$ is equivalent to the following bilinear fractional programming problem:

$$
\begin{array}{|cl}
\text { maximize } & \frac{\sigma_{0}-\sum_{j=1}^{n_{1}}\left(q_{j}+q_{j}^{\prime} y_{j}\right) x_{j}+\sum_{k=1}^{n_{2}}\left(Q_{k}+Q_{k}^{\prime} Y_{k}\right) X_{k}}{} \\
& \pi_{0}-\sum_{j=1}^{n_{1}}\left(r_{j}+r_{j}^{\prime} y_{j}\right) x_{j}+\sum_{k=1}^{n_{2}}\left(R_{k}+R_{k}^{\prime} Y_{k}\right) X_{k} \\
\text { subject to } & \tilde{\beta}_{i} \leq \sum_{j=1}^{n_{1}}\left(g_{i j}+g_{i j}^{\prime} y_{j}\right) x_{j}+\sum_{k=1}^{n_{2}}\left(G_{i k}+G_{i k}^{\prime} Y_{k}\right) X_{k} \leq \tilde{a}_{i}, i=1, \ldots, m_{1} \\
& \sum_{j=1}^{n_{1}} a_{l j} x_{j}+\sum_{k=1}^{n_{2}} a_{l k}^{\prime} X_{k} \leq a_{l 0}, l=1, \ldots, m_{2} \\
& 0 \leq x_{j} \leq u_{j}, y_{j}^{0} \leq y_{j} \leq y_{j}^{1}, j=1, \ldots n_{1} \\
& 0 \leq X_{k} \leq C_{k}, Y_{k}^{0} \leq Y_{k} \leq Y_{k}^{1}, k=1, \ldots, n_{2}
\end{array}
$$


Let us now turn to the partial optimization model, the main topic of this paper in which the difference of a giren objective, say $O_{1}$ associated with the bundle of assets $\left(X_{1}, \ldots, X_{n_{2}}\right)$ purchased from the market and $\left(x_{1} \ldots \ldots x_{n_{1}}\right)$ sold to the market subject to the same constraints as (18) (See Fig 1(b)). This means that we evaluate the objective and constraints relative to the total portfolio owned by an investor after the transaction except the objective $O_{1}$.

The problem can now be formulated as follows:

$$
\begin{aligned}
& \begin{array}{|ll}
\text { maximize } & \frac{\sum_{k=1}^{n_{2}}\left(Q_{k}+Q_{k}^{\prime} Y_{k}\right) x_{k}}{\sum_{k=1}^{n_{2}}\left(R_{k}+R_{k}^{\prime} Y_{k}\right) x_{k}}-\frac{\sum_{j=1}^{n_{1}}\left(q_{j}+q_{j}^{\prime} y_{j}\right) x_{j}}{\sum_{j=1}^{n_{1}}\left(r_{j}+r_{j}^{\prime} y_{j}\right) x_{j}} \\
\text { subject to } & \tilde{3}_{i} \leq \sum_{k=1}^{n_{2}}\left(G_{i k}+G_{i k}^{\prime} Y_{k}\right) X_{k}+\sum_{j=1}^{n_{1}}\left(g_{i j}+g_{i j}^{\prime} y_{j}\right) x_{j} \leq \tilde{a}_{i}, i=1 \ldots, m_{1}
\end{array} \\
& \sum_{j=1}^{n_{1}} a_{l j} x_{j}+\sum_{k=1}^{n_{2}} a_{l k}^{\prime} x_{k} \leq a_{l 0}, l=1 \ldots m_{2} \\
& 0 \leq x_{j} \leq u_{j}, y_{j}^{0} \leq y_{j} \leq y_{j}^{1}, j=1, \ldots, n_{1} \\
& 0 \leq \mathrm{I}_{k} \leq U_{k}, Y_{k}^{0} \leq Y_{k} \leq Y_{k}^{1}, k=1, \ldots, n_{2}
\end{aligned}
$$

where as before, we will assume that the divisors of the objective functions are positive for all feasible solutions. Also, we assume that the divisors in the objective function are of similar magnitude, so that it is meaningful to compare the difference. A typical example is the one explained is Section 5 in which the variables $\left(x_{1}, \ldots, x_{n_{1}}\right)$ and $\left(X_{1}, \ldots, X_{n_{2}}\right)$ are constrained so that

$$
\sum_{k=1}^{n_{2}} Y_{k} X_{k}=\sum_{j=1}^{n_{1}} y_{j} x_{j}+C
$$

This means that the total amount of cash spent for purchasing the portfolio $\left(X_{1}, \ldots, X_{n_{2}}\right)$ is the total amount of cash from the sale of the portfolio $\left(x_{1}, \ldots, x_{n_{1}}\right)$ plus the income $C$ from the coupon payment during the given period. It then follows that divisors of performance index (a) $\sim(f)$ associated with portfolios $x$ and $X$ are of the similar magnitude.

\section{Algorithms for Solving Total and Partial optimization Models}

Let us now discuss the algorithms for calculating globally optimal solutions of the problems (18) and (19). The first step to introduce auxiliary variables:

$$
\begin{aligned}
& z_{j}=y_{j} x_{j}, j=1, \ldots, n_{1} \\
& Z_{k}=Y_{k} X_{k}, k=1, \ldots, n_{2}
\end{aligned}
$$

Then the constraints $y_{j}^{0} \leq y_{j} \leq y_{j}^{1}$ and $Y_{k}^{0} \leq Y_{k} \leq Y_{k}^{1}$ are equivalent to the following conditions:

$$
\begin{aligned}
& y_{j}^{0} x_{j} \leq z_{j} \leq y_{j}^{1} x_{j}, j=1, \ldots, n_{1} \\
& Y_{k}^{0} \mathrm{X}_{k} \leq Z_{k} \leq Y_{k}^{1} \mathrm{I}_{k}, k=1, \ldots n_{2}
\end{aligned}
$$


Thus the problems (18) is reformulated as a linear fractional programming problem:

$$
\begin{array}{|ll}
\operatorname{maximize} & \frac{\sigma_{0}-\sum_{j=1}^{n_{1}}\left(q_{j} x_{j}+q_{j}^{\prime} \tilde{\sim}_{j}\right)+\sum_{k=1}^{n_{2}}\left(Q_{k} X_{k}+Q_{k}^{\prime} Z_{k}\right)}{\pi_{0}-\sum_{j=1}^{n_{1}}\left(r_{j} x_{j}+r_{j}^{\prime} z_{j}\right)+\sum_{k=1}^{n_{2}}\left(R_{k} X_{k}+R_{k}^{\prime} Z_{k}\right)} \\
\text { subject to } & \tilde{j}_{i} \leq \sum_{j=1}^{n_{1}}\left(g_{i j} x_{j}+g_{i j}^{\prime} \tilde{\sim}_{j}\right)+\sum_{k=1}^{n_{2}}\left(G_{i k} X_{k}+G_{i k}^{\prime} Z_{k}\right) \leq \tilde{a}_{i}, i=1 \ldots, m_{1} \\
& \sum_{j=1}^{n_{1}} a_{l j} x_{j}+\sum_{k=1}^{n_{2}} a_{l k}^{\prime} X_{k} \leq a_{l 0}, l=1, \ldots, m_{2} \\
& 0 \leq x_{j} \leq u_{j} \cdot y_{j}^{0} x_{j} \leq z_{j} \leq y_{j}^{1} x_{j}, j=1, \ldots, n_{1} \\
& 0 \leq X_{k} \leq C_{k} . Y_{k}^{0} X_{k} \leq Z_{k} \leq Y_{k}^{1} X_{k}, k=1, \ldots, n_{2}
\end{array}
$$

which can be solved by standard methods [2]. Also, the problem (19) is reformulated as follows:

$$
\begin{array}{|cl}
\operatorname{maximize} & \frac{\sum_{k=1}^{n_{2}}\left(Q_{k} X_{k}+Q_{k}^{\prime} Z_{k}\right)}{\sum_{k=1}^{n_{2}}\left(R_{k} X_{k}+R_{k}^{\prime} Z_{k}\right)}-\frac{\sum_{j=1}^{n_{1}}\left(q_{j} x_{k}+q_{j}^{\prime} z_{j}\right)}{\sum_{j=1}^{n_{1}}\left(r_{j} x_{j}+r_{j}^{\prime} \tilde{\sim}_{j}\right)} \\
\text { subject to } & \tilde{j}_{i} \leq \sum_{j=1}^{n_{1}}\left(g_{i j} x_{j}+g_{i j}^{\prime} \tilde{z}_{j}\right)+\sum_{k=1}^{n_{2}}\left(G_{i k} \mathrm{I}_{k}+G_{i k}^{\prime} Z_{k}\right) \leq \tilde{a}_{i}, i=1, \ldots, m_{1} \\
& \sum_{j=1}^{n_{1}} a_{l j} x_{j}+\sum_{k=1}^{n_{2}} a_{l k}^{\prime} X_{k} \leq a_{l 0}, l=1, \ldots, m_{2} \\
& 0 \leq x_{j} \leq u_{j}, y_{j}^{0} x_{j} \leq z_{j} \leq y_{j}^{1} x_{j}, j=1, \ldots, n_{1} \\
& 0 \leq X_{k} \leq U_{k}, Y_{k}^{0} X_{k} \leq Z_{k} \leq Y_{k}^{1} X_{k}, k=1, \ldots, n_{2}
\end{array}
$$

This looks much simpler than (19) but it cannot be solved by standard nonlinear programming algorithms [1] since the objective function is not (quasi-)convex. Fortunately however, we will show in the sequel that a global optimum of this problem can be obtained by a variant of parametric simplex algorithm dereloped by the authors [9].

To explain the algorithm, let us first introduce a new set of variables:

$$
v_{j}= \begin{cases}x_{k}, & j=1, \ldots, n_{1} \\ z_{j-n_{1}}, & j=n_{1}+1, \ldots 2 n_{1} \\ X_{j-2 n_{1}}, & j=2 n_{1}+1, \ldots, 2 n_{1}+n_{2} \\ Z_{j-2 n_{1}-n_{2},} & j=2 n_{1}+n_{2}+1, \ldots, 2 n_{1}+2 n_{2}\end{cases}
$$

and denote (26) as follows:

$$
\begin{array}{|ll}
\text { maximize } & \frac{\sum_{j=1}^{n} \rho_{j}^{\prime} v_{j}}{} \\
& \sum_{j=1}^{n} \rho_{j} v_{j}-\frac{\sum_{j=1}^{n} \sigma_{j}^{\prime} v_{j}}{\sum_{j=1}^{n} \sigma_{j} v_{j}} \\
\text { subject to } & \sum_{j=1}^{n} a_{i j} v_{j} \leq a_{i 0}, i=1, \ldots, m \\
& 0 \leq v_{j} \leq v_{j}^{0}: j=1, \ldots, n
\end{array}
$$

where $n=2 n_{1}+2 n_{2}$. Let

$$
w=\frac{1}{\sum_{j=1}^{n} \sigma_{j} \imath_{j}^{\prime}}
$$


By assumption. we have $w>0$ for all $v_{j}$ s satisfying the constraints of (26). Therefore the problem is equiralent to

$$
\begin{array}{|ll}
\text { maximize } & \frac{\sum_{j=1}^{n} \rho_{j}^{\prime} v_{j} \cdot w}{\sum_{j=1}^{n} \rho_{j} v_{j} \cdot w}-\sum_{j=1}^{n} \sigma_{j}^{\prime} v_{j} \cdot w \\
\text { subject to } & \sum_{j=1}^{n} a_{i j} v_{j} w \leq a_{i 0} w, i=1, \ldots, m \\
& 0 \leq v_{j} w^{\prime} \leq v_{j}^{0} u^{\prime}, j=1, \ldots, n \\
& \sum_{j=1}^{n} \sigma_{j} v_{j} w=1
\end{array}
$$

Let us define

$$
V_{j}=v_{j} \cdot u^{\prime}, j=1, \ldots, n
$$

then $(30)$ is equivalent to

$$
\begin{aligned}
\text { subject to } & \sum_{j=1}^{n} a_{i j} V_{j}-a_{i 0} w \leq 0, i=1, \ldots, m \\
& \sum_{j=1}^{n} \sigma_{j} V_{j}=1 \\
& 0 \leq V_{j} \leq v_{j}^{0} w, j=1, \ldots, n
\end{aligned}
$$

Finally, let

$$
\begin{aligned}
S=\left\{\left(V_{1}, \ldots, V_{n}, w\right) \mid\right. & \mid \sum_{j=1}^{n} a_{i j} V_{j}-a_{i 0} w \leq 0, \\
& \left.\sum_{j=1}^{n} \sigma_{j} V_{j}=1,0 \leq V_{j} \leq v_{j}^{0} w, \quad j=1, \ldots, n\right\}
\end{aligned}
$$

and let

$$
\xi=\sum_{j=1}^{n} \rho_{j} V_{j}
$$

also let

$$
\begin{aligned}
& \xi_{\max }=\max \left\{\sum_{j=1}^{n} \rho_{j} V_{j} \mid\left(V_{1}, \ldots, V_{n}, w^{\prime} \in S\right\}\right. \\
& \xi_{\min }=\min \left\{\sum_{j=1}^{n} \rho_{j} V_{j} \mid\left(V_{1}, \ldots, V_{n}, w^{\prime}\right) \in S\right\}
\end{aligned}
$$


Then (32) can be put in the following form:

$$
\begin{array}{|ll}
\text { maximize } & \frac{1}{\xi} \sum_{j=1}^{n} \rho_{j}^{\prime} V_{j}-\sum_{j=1}^{n} \sigma_{j}^{\prime} \boldsymbol{V}_{j} \\
\text { subject to } & \sum_{j=1}^{n} \rho_{j} V_{j}=\xi \\
& \sum_{j=1}^{n} a_{i j} V_{j}-a_{i 0} w \leq 0, i=1, \ldots, m \\
& \sum_{j=1}^{n} \sigma_{j} V_{j}=1 \\
& 0 \leq V_{j} \leq v_{j}^{0} u, j=1, \ldots, n \\
& \xi_{\min } \leq \xi \leq \xi_{\max }
\end{array}
$$

Let us note that the problem (3T) reduces to a linear programming problem if we fix the value of $\xi$. Let $\xi_{0} \in\left[\xi_{\min }, \xi_{\max }\right]$ and consider a linear programming problem.

$$
\begin{array}{|ll}
\text { maximize } & \sum_{j=1}^{n}\left(\frac{\rho_{j}^{\prime}}{\xi_{0}}-\sigma_{j}^{\prime}\right) V_{j} \\
\text { subject to } & \sum_{j=1}^{n} \rho_{j} V_{j}=\xi_{0} \\
& \sum_{j=1}^{n} a_{i j} V_{j}-a_{i 0} w \leq 0, i=1, \ldots, m \\
& \sum_{j=1}^{n} \sigma_{j} V_{j}=1 \\
& 0 \leq V_{j} \leq v_{j}^{0} w, j=1, \ldots, n
\end{array}
$$

Let $B$ be an optimal basic solution associated with (38). This basis remains optimal for all values of $\xi$ in the interval $[\xi, \bar{\xi}]$ such that the primal feasibility and dual feasibility are maintained. Thus we can apply a parametric simplex algorithm for solving (37) analytically for all $\xi$ in the interval $\left[\xi_{\min }, \xi_{\max }\right]$. Let us note that the objective function has a simple form $a \xi+b \frac{1}{\xi}+c$ in each subinterval, so that we can calculate a global optimum of $(37)$ in finitely many steps. Readers not familiar with parametric simplex algorithms are referred to either [9] or to a standard text of linear programming[3].

\section{Results of Numerical Experiments}

We will present some preliminary results of numerical simulation on the partial optimization model using the available data of about 90 government bonds circulated in the market. We adopted the arerage effective yield as an objective to be optimized and solved the following problem:

$$
\begin{array}{|ll}
\text { maximize } & \nu(X)-\nu(x) \\
\text { subject to } & d(W)=d_{0} \\
& C(W) \geq C_{0} \\
& \sum_{k=1}^{n_{2}} P_{k} X_{k}=\sum_{j=1}^{n_{1}} p_{j} x_{j}+C \\
& 0 \leq x_{j} \leq u_{j}, j=1, \ldots, n_{1} \\
& 0 \leq X_{k} \leq U_{k}, k=1, \ldots, n_{2}
\end{array}
$$

where $\nu(X)$ and $\nu(x)$ are arerage effective yield of the bundle of assets $X$ and $x$, respectively. Also $d\left(W^{*}\right)$ and $C\left(W^{\circ}\right)$ are arerage duration and average convexity of the portfolio $Y$ owned 
by an investor after the transaction, i.e..

$$
W_{j}= \begin{cases}u_{j}-x_{j}, & j=1, \ldots n_{1} \\ X_{j-n_{1}}, & j=n_{1}+1, \ldots, n_{1}+n_{2}\end{cases}
$$

while $d_{0}$ and $C_{0}$ are the duration and convexity of the index portfolio. respectively. The third set of constraints means that we purchase the portfolio $X$ by the available fund from the sale of portfolio $x$ plus the amount of coupon payment $C$ during the given period. Also, we assumed that the price adjustment coefficients are all zeros, i.e., that all the prices are held constant.

The upper bound $t_{k}$ on $X_{k}$ is chosen to be $5 \%$ of the total amount of the bond available in the market. Also, we assume that all bonds in the portfolio can be sold to the market. We started from a unit value portfolio consisting of a single bond and repeated solving the problem (39) for thirty nine periods using $W$ as the starting portfolio in the next period.

We repeated this process eighty two times by choosing all available government bonds as starting "single bond" portfolios.

Figure 2 shows the ralues of the best and the worst one among these eighty two portfolios in terms of the ralues at the end of the thirty nine periods horizon. The best one labeled No. 211 outperforms the Yamaichi index while the worst one labeled No. 111 is a little behind the index. We see from this figure that we can keep track of the index portfolio very well by using our model. Also Figure 3 shows the monthly rates of return of the calculated portfolio and the index portfolio. Let us note that the portfolio consists of at most seven bonds, which is rery desirable from the viewpoint of bond managers. Also, the computation time for solving the problem (39) is about 5 seconds on SONY NWS-3800 Workstation.

\section{Conclusions and the Future Direction of Research}

We showed in this paper that both partial optimization models and total optimization models proposed in [7] for bond portfolio management of institutional investors can be solved to optimality in an efficient way by using a global optimization algorithm developed by one of the authors[9]. In the last several years, a simplified version of the total optimization model has been used by several institutional investors. Instead, a partial optimization model was not used because of computational difficulty. However, it is now ready for use for the first time in a real transaction environment. As stated in the Introduction, there has been a significant needs of bond managers of institutional investors for a partial optimization model since only a fraction, typically less than one tenth of the total assets is subject to transaction. The partial optimization model meets the request of such bond managers to evaluate their transaction.

Due to the limited availability of the real market data, we could not conduct an extensive test to fully demonstrate the potential power of this model. Therefore we applied a partial optimization model to the index tracking of government bonds, by using the available market data. The result shors that this model generates a remarkably good result both in terms of computational efficiency and quality of the resulting portfolio. To demonstrate the usefulness of our approach, a more extensive simulation has to be conducted, whose results as well as further improvements of the model will be discussed elsewhere.

\section{Acknowledgements}

The first author acknowledges the generous support of the Dai-ichi Life Insurance Co. and the Toyo Trust and Banking Co. Ltd.. 


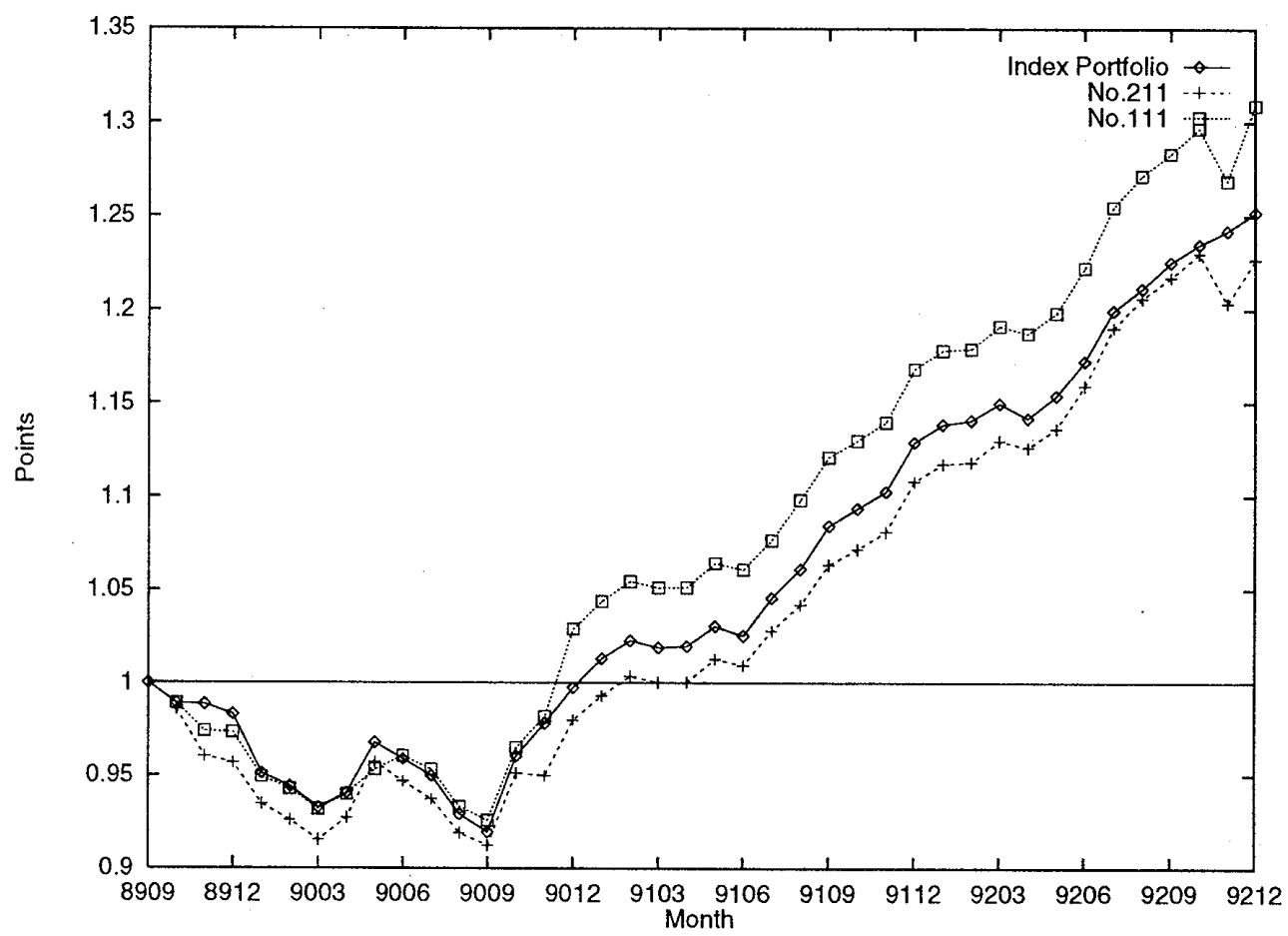

Figure 2: Values of The Portfolios

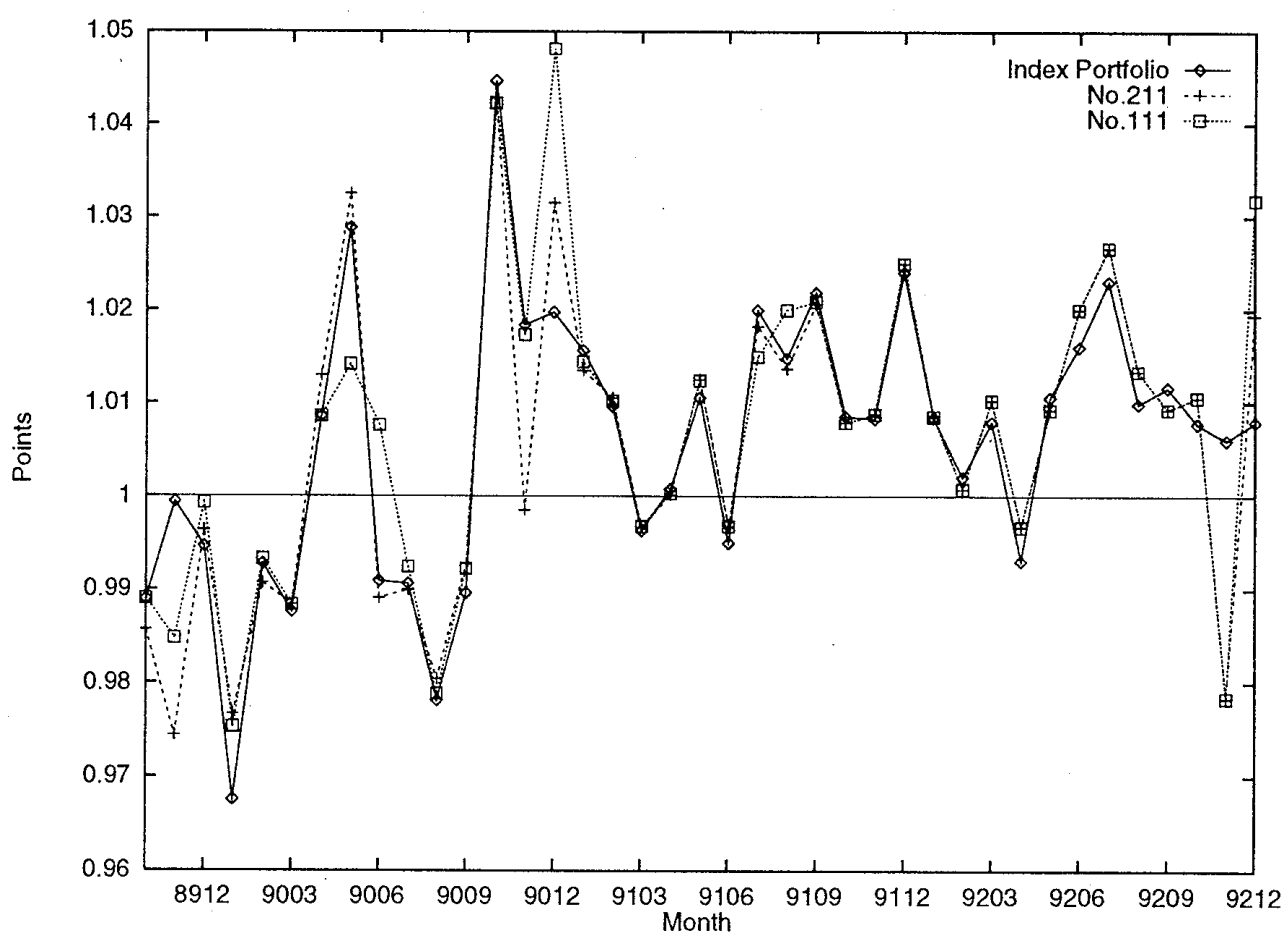

Figure 3: Monthly Rate of Returns 


\section{References}

[1] Avriel. M., Nonlinier Programming, Prentice-Hall, 1976.

[2] Charnes, A. and Cooper. W.W., "Programming with Linear Fractional Functions", Naval Research Logistics Quarterly, 9(1962) 181 186.

[3] Chvátal, V., Linear Programming, Freeman and Co.,1983.

[4] Elton, E.J. and Gruber, N.J.. Modern Portfolio Theory and Investment Analysis (4th ed.) John Wiley \& Sons, Inc., 1991.

[5] Fong, G.H. and Fabozzi, F.J., Fixed Income Portfolio Management. Dow Jones, Irwin, Inc., 1985.

[6] Horst, R. and Tuy H., Global Optimization, Springer Verlag, 1991.

[7] Konno, H. and Inori, M., "Bond Portfolio Optimization by Bilinear Fractional Programming", J. of the Operations Research Society of Japan, 32 (1989) 143-158.

[8] Konno, H. and Takase, T., "Estimating the Term Structure of Interest Rates by Constrained Least Square Approach", to appear in Financial Engineering and Japanese Markets.

[9] Konno, H., Yajima, Y. and Matsui, "Parametric Simplex Algorithms for Solving a Special Class of Nonconvex Minimization Problems", J. of Global Optimization, 1 (1991) 65-81.

[10] Lasdon. L., Optimization Theory for Large Systems, Macmillian Co., 1970.

[11] Luenberger, D.G., Introduction to Linear and Nonlinear Programming. (2nd edition). Addison-Wesley, 1984.

Hiroshi KONNO:

Department of Industrial Engineering and Management

Tokyo Institute of Technology

2-12-1 Oh-okayama, Meguro-ku

Tokyo 152, Japan

The second author Hidetoshi Watabe is currently at NTT DATA CORPORATION. 